\title{
Hierarchical fixed points of strictly pseudo contractive mappings for variational inequality problems
}

\author{
Tanom Chamnarnpan ${ }^{1}$, Nopparat Wairojjana ${ }^{1,2}$ and Poom Kumam ${ }^{1 *}$
}

\begin{abstract}
In this paper, we introduce a new iterative scheme that converges strongly to a common fixed point of a countable family of strictly pseudo-contractive mappings in a real Hilbert space which is also a solution of variational inequality problem related to quadratic minimization problems. Our results extend ones of Yao et al. [Math. and Comput. Modell. 52(9-10):1697-1705, 2010], Gu et al. [J. Appl. Math. 2011:17 p., 2011] and some authors.
\end{abstract}

Keywords: Strong convergence; Strictly pseudo-contractive mapping; Hilbert spaces; Variational inequality; Hierarchical fixed point problem

\section{Introduction}

Throughout this paper, we assume that $H$ is a real Hilbert space with inner product and norm denoted by $\langle\cdot, \cdot\rangle$ and $\|\cdot\|$, respectively and let $C$ is a nonempty closed and convex subset of $H$. A mapping $f: C \rightarrow H$ is called a contraction on $C$ if there exists a constant $\rho \in[0,1)$ such that

$$
\|f(x)-f(y)\| \leq \rho\|x-y\|, \quad \forall x, y \in C .
$$

A mapping $T: C \rightarrow H$ is called a nonexpansive on $C$ if

$$
\|T x-T y\| \leq\|x-y\|, \quad \forall x, y \in C .
$$

A mapping $M: H \rightarrow H$ is called a strongly monotone operator with coefficient $\alpha$ if there exists a constant $\alpha>0$ such that

$$
\langle x-y, M x-M y\rangle \geq \alpha\|x-y\|^{2}, \quad \forall x, y \in H
$$

and $M$ is called a monotone operator if

$$
\langle x-y, M x-M y\rangle \geq 0, \quad \forall x, y \in H .
$$

It is well known that the mapping $(I-T)$ is a monotone operator, if $T$ is a nonexpansive mapping and $I$ is a identity mapping.

\footnotetext{
*Correspondence: poom.kum@kmutt.ac.th

1 Department of Mathematics, Faculty of Science, King Mongkut's University of Technology Thonburi (KMUTT), 126 Pracha Uthit Road, Bang Mod, Thrung Khru, Bangkok 10140, Thailand

Full list of author information is available at the end of the article
}

A mapping $T: C \rightarrow H$ is called $k$-strict pseudocontraction if there exists a constant $k \in[0,1)$ such that

$$
\|T x-T y\|^{2} \leq\|x-y\|^{2}+k\|(I-T) x-(I-T) y\|^{2}, \quad \forall x, y \in C .
$$

If there exists a point $x \in C$ such that $x=T x$, then $x$ is said to be fixed point of $T$. We denote the set of all fixed pionts of $T$ by $F(T)$. It is well known that $F(T)$ is closed and convex if $T$ is nonexpansive.

Note that the class of $k$-strict pseudo-contraction mappings includes the class of nonexpansive mappings on $C$ as a subclass. i.e., $T$ is nonexpansive if and only if $T$ is 0 -strict pseudo-contraction. Recently, many authors have been devoting the studies on the problems of finding fixed points for $k$-strict pseudo-contraction mappings; see Acedo and Xu (2007); Cho et al. (2009); Jung (2010); Jung (2011); Zhou (2008) and the references therein.

A variational inequality in a real Hilbert space $H$ is formulated as finding a point $x^{*} \in C$ such that

$$
\left\langle F x^{*}, x-x^{*}\right\rangle \geq 0, \quad \forall x \in C,
$$

where $F: C \rightarrow H$ is a nonlinear mapping. We denote the set of solution of $(1)$ by $V I(C, F)$. If $F$ is a monotone operator, then (1) is also known as a monotone variational inequality.

For given nonlinear operators $F, g$, we consider the problem of finding $u \in H$ such that

$$
\langle F(u), g(v)-g(u)\rangle \geq 0, \quad \forall g(v), g(u) \in C
$$

\section{是 Springer}


which is known as the general variational inequality prob$l e m$. For given nonlinear operators $F, g$, $h$, we consider the problem of finding $u \in H: h(u) \in C$ such that

$$
\langle F(u), g(v)-h(u)\rangle \geq 0, \quad \forall g(v) \in C
$$

which is called the extended general variational inequality.

The variational inequalities have been studied widely and are being used as a mathematical programming tool in modeling a wide class of problems arising in several branches of pure and applied sciences; see Baiocchi and Capelo (1984); Giannessi and Maugeri (1995); Kinderlehrer and Stampacchia (1980). For general variational inequalities and extended general variational inequalities, we can refer Noor $(2004,2009)$; Noor et al. (2012a,2012b) and references therein.

It is well-known that the variational inequality (1) is equivalent to the fixed point equation

$$
x^{*}=P_{C}\left[(I-\gamma F) x^{*}\right]
$$

where $\gamma>0$ and $P_{C}$ is the metric projection of $H$ onto $C$ which assigns, to each $x \in H$, the unique point in $C$, denoted $P_{C}[x]$, such that

$$
\left\|x-P_{C}[x]\right\|=\inf \{\|x-y\|: y \in C\} .
$$

Therefore, fixed point algorithms can be applied to solve variational inequalities.

The following problem is called a hierarchical fixed point problem: Find $x^{*} \in F(T)$ such that

$$
\left\langle x^{*}-S x^{*}, x-x^{*}\right\rangle \geq 0, \quad \forall x \in F(T) .
$$

where $S: C \rightarrow H$ be a mapping. It is known that the hierarchical fixed point problem (5) links with some monotone variational inequalities and convex programming problems; see Gu et al. (2011); Yao et al. (2010).

In order to solve the hierarchical fixed point problem (5), Moudafi (2007) intoduced the following KrasnoselskiMann algorithm:

$$
x_{n+1}=\left(1-\alpha_{n}\right) x_{n}+\alpha_{n}\left(\beta_{n} S x_{n}+\left(1-\beta_{n}\right) T x_{n}\right),
$$

where $S, T: C \rightarrow C$ are two nonexpansive mappings, $\left\{\alpha_{n}\right\}$ and $\left\{\beta_{n}\right\}$ are two sequences in $(0,1)$. Then he showed that $\left\{x_{n}\right\}$ converges weakly to a fixed point of $T$ which is a solution of problem (5). For obtaining a strong convergence result, in Mainge and Moudafi (2007) and Marino and Xu (2011) introduced the following algorithm:

$$
x_{n+1}=\left(1-\alpha_{n}\right) f\left(x_{n}\right)+\alpha_{n}\left(\beta_{n} S x_{n}+\left(1-\beta_{n}\right) T x_{n}\right),
$$

where $f: C \rightarrow C$ is a contraction mapping, $S$ and $T$ : $C \rightarrow C$ are two nonexpansive mappings, $\left\{\alpha_{n}\right\}$ and $\left\{\beta_{n}\right\}$ are two sequences in $(0,1)$. Then they showed that $\left\{x_{n}\right\}$ converges strongly to a fixed point of $T$ which is a solution of problem (5).
On the other hand, Cianciaruso et al. (2009) introduced a two step algorithm to solve the problem (5) as follows:

$$
\left\{\begin{array}{l}
y_{n}=\beta_{n} S x_{n}+\left(1-\beta_{n}\right) x_{n} \\
x_{n+1}=\alpha_{n} f\left(x_{n}\right)+\left(1-\alpha_{n}\right) T y_{n}
\end{array}\right.
$$

where $f: C \rightarrow C$ is a contraction mapping, $S$ and $T: C \rightarrow C$ are two nonexpansive mappings, $\left\{\alpha_{n}\right\}$ and $\left\{\beta_{n}\right\}$ are two sequences in $(0,1)$. Under some certain restrictions on parameters, the authors proved the sequence $\left\{x_{n}\right\}$ generated by (8) converges strongly to $x^{*} \in F(T)$, which is a unique solution of the following variational inequality:

$$
\left\langle(I-f) x^{*}, x-x^{*}\right\rangle \geq 0, \quad \forall x \in F(T) .
$$

By changing the restrictions on parameters, the authors obtained another result on the iterative scheme (8) , i.e., the sequence $\left\{x_{n}\right\}$ generated by (8) converges strongly to $x^{*} \in F(T)$, which is a unique solution of the following variational inequality:

$$
\left\langle\frac{1}{\tau}(I-f) x^{*}+(I-S) x^{*}, x-x^{*}\right\rangle \geq 0, \quad \forall x \in F(T),
$$

where $\tau \in(0, \infty)$ is a constant.

In 2010, Yao et al. (2010) modified the two step algorithm (8) to extend Range of $f$ from $C$ to $H$ by using the metric projection of $H$ onto $C$. They introduced the following iterative scheme:

$$
\left\{\begin{array}{l}
y_{n}=\beta_{n} S x_{n}+\left(1-\beta_{n}\right) x_{n}, \\
x_{n+1}=P_{C}\left[\alpha_{n} f\left(x_{n}\right)+\left(1-\alpha_{n}\right) T y_{n}\right]
\end{array}\right.
$$

where $f: C \rightarrow H$ is a contraction mapping, $S$ and $T: C \rightarrow C$ are two nonexpansive mappings, $\left\{\alpha_{n}\right\}$ and $\left\{\beta_{n}\right\}$ are two sequences in $(0,1)$. The authors proved the sequence $\left\{x_{n}\right\}$ generated by (11) converges strongly to $x^{*} \in$ $F(T)$, which is a unique solution of one of the variational inequalities (9) and (10).

In 2011, Gu et al. (2011) introduced the following iterative algorithm:

$$
\left\{\begin{array}{l}
y_{n}=P_{C}\left[\beta_{n} S x_{n}+\left(1-\beta_{n}\right) x_{n}\right], \\
x_{n+1}=P_{C}\left[\alpha_{n} f\left(x_{n}\right)+\sum_{i=1}^{n}\left(\alpha_{i-1}-\alpha_{i}\right) T_{i} y_{n}\right], \quad \forall n \geq 1,
\end{array}\right.
$$

where $f: C \rightarrow H$ is a contraction mapping, $S: C \rightarrow H$ is a nonexpansive mapping, $\left\{T_{i}\right\}_{i=1}^{\infty}: C \rightarrow C$ is a countable family of nonexpansive mappings, $\alpha_{0}=1,\left\{\alpha_{n}\right\}$ and $\left\{\beta_{n}\right\}$ are two sequences in $(0,1)$. The authors proved the sequence $\left\{x_{n}\right\}$ generated by (12) converges strongly to $x^{*} \in$ $F(T)$, which is a unique solution of one of the variational inequalities (9) and (10). 
In this paper, motivated and inspired by the results of $\mathrm{Gu}$ et al. (2011), we introduce and study the following iterative scheme:

$$
\left\{\begin{array}{l}
y_{n}=P_{C}\left[\beta_{n} S x_{n}+\left(1-\beta_{n}\right) x_{n}\right], \\
x_{n+1}=P_{C}\left[\alpha_{n} f\left(x_{n}\right)+\sum_{i=1}^{n}\left(\alpha_{i-1}-\alpha_{i}\right) V_{i} y_{n}\right], \quad \forall n \geq 1,
\end{array}\right.
$$

where $V_{i}=k_{i} I+\left(1-k_{i}\right) T_{i}$ and $\left\{T_{i}\right\}_{i=1}^{\infty}: C \rightarrow C$ is a countable family of $k_{i}$-strict pseudo-contraction mappings. Under some certain condition on parameters, we first prove that the sequence $\left\{x_{n}\right\}$ generated by (13) converges strongly to $x^{*} \in \cap_{i=1}^{\infty} F\left(T_{i}\right)$ which is a unique solution of the following variational inequality:

$$
\left\langle(I-f) x^{*}, x-x^{*}\right\rangle \geq 0, \quad \forall x \in \cap_{i=1}^{\infty} F\left(T_{i}\right) .
$$

By changing the restrictions on parameters, we also prove that the sequence $\left\{x_{n}\right\}$ generated by (13) converges strongly to $x^{*} \in \cap_{i=1}^{\infty} F\left(T_{i}\right)$, which is a unique solution of the following variational inequality:

$$
\left\langle\frac{1}{\tau}(I-f) x^{*}+(I-S) x^{*}, x-x^{*}\right\rangle \geq 0, \quad \forall x \in \cap_{i=1}^{\infty} F\left(T_{i}\right),
$$

where $\tau \in(0, \infty)$ is a constant. It is easy to see that, if $k_{i}=0$ for each $i \geq 1$, then our algorithm (13) is reduced to algorithm (12) of $\mathrm{Gu}$ et al. Also our results extend the corresponding one of Yao et al. (2010); Xu (2004); Cianciaruso et al. (2009); Moudafi (2000) and Gu et al. (2011) from the countable family of nonexpansive mappings to more general the countable family of strictly pseudo contraction mappings.

\section{Preliminaries}

This section collects some lemma which be use in the proofs for the main results in the next section. Some of them are known; others are not hard to derive.

We will use the following notation:

(i) $\rightarrow$ for strong convergence and $\rightarrow$ for weak convergence.

(ii) $\omega_{w}\left(x_{n}\right)=\left\{x: x_{n_{i}} \rightarrow x\right\}$ denotes the weak $\omega$-limit set of $\left\{x_{n}\right\}$.

Lemma 1. Browder (1976) Let $H$ be a Hilbert space, $C$ is a closed convex subset of $H$ and $T: C \rightarrow C$ be a nonexpansive mapping with $F(T) \neq \emptyset$. If $\left\{x_{n}\right\}$ is a sequence in $C$ weakly converging to $x$ and if $\left\{(I-T) x_{n}\right\}$ converges strongly to $y$, then $(I-T) x=y$; in particular, if $y=0$ then $x \in F(T)$.

Lemma 2. Acedo and $\mathrm{Xu}$ (2007) Let $C$ be a nonempty closed convex subset of a real Hilbert space H. If $T: C \rightarrow C$ is a $k$-strict pseudo-contraction, then the mapping $I-T$ is demiclosed at 0 . That is, if $\left\{x_{n}\right\}$ is a sequence in $C$ weakly converging to $x$ and $\left\{(I-T) x_{n}\right\}$ converges strongly to 0 , then $(I-T) x=0$.

Lemma 3. Let $x \in H$ and $z \in C$ be any points. Then we have the following:

1. That $z=P_{C}[x]$ if and only if there holds the relation:

$$
\langle x-z, y-z\rangle \leq 0, \quad \forall y \in C .
$$

2. That $z=P_{C}[x]$ if and only if there holds the relation:

$$
\|x-z\|^{2} \leq\|x-y\|^{2}-\|y-z\|^{2}, \forall y \in C .
$$

3. There holds the relation:

$\left\langle P_{C}[x]-P_{C}[y], x-y\right\rangle \geq\left\|P_{C}[x]-P_{C}[y]\right\|^{2}, \forall x, y \in H$.

Consequently, $P_{C}$ is nonexpansive and monotone.

Lemma 4. Marino and $\mathrm{Xu}$ (2006) Let H be a Hilbert space, $C$ be a closed convex subset of $H, f: C \rightarrow H$ be a contraction with coefficient $0<\rho<1$ and $T: C \rightarrow C$ be a nonexpansive mapping. Then, for $0<\gamma<\bar{\gamma} / \rho$, for $x, y \in C$,

1. the mapping $(I-f)$ is strongly monotone with coefficient $(1-\rho)$ that is

$$
\langle x-y,(I-f) x-(I-f) y\rangle \geq(1-\rho)\|x-y\|^{2} .
$$

2. the mapping $(I-T)$ is monotone, that is

$$
\langle x-y,(I-T) x-(I-T) y\rangle \geq 0 .
$$

Lemma 5. $\mathrm{Xu}$ (2002) Assume that $\left\{a_{n}\right\}$ is a sequence of nonnegative numbers such that

$$
a_{n+1} \leq\left(1-\gamma_{n}\right) a_{n}+\delta_{n}, \quad \forall n \geq 0,
$$

where $\left\{\gamma_{n}\right\}$ is a sequence in $(0,1)$ and $\left\{\delta_{n}\right\}$ is a sequence in $\mathcal{R}$ such that

1. $\sum_{n=1}^{\infty} \gamma_{n}=\infty$,

2. $\limsup _{n \rightarrow \infty} \frac{\delta_{n}}{\gamma_{n}} \leq 0$ or $\sum_{n=1}^{\infty}\left|\delta_{n}\right|<\infty$.

Then $\lim _{n \rightarrow \infty} a_{n}=0$.

Lemma 6. Acedo and $\mathrm{Xu}$ (2007) Let $C$ be a closed convex subset of $H$. Let $\left\{x_{n}\right\}$ be a bounded sequence in H. Assume that

(1) The weak $\omega$-limit set $\omega_{w}\left(x_{n}\right) \subset C$,

(2) For each $z \in C, \lim _{n \rightarrow \infty}\left\|x_{n}-z\right\|$ exists.

Then $\left\{x_{n}\right\}$ is weakly convergent to a point in $C$.

Lemma 7. Zhou (2008) Let $H$ be a real Hilbert space, $C$ be a closed and convex subset of $H$, and $T$ be a $k$-strict pseudocontraction mapping on $C$, then $F(T)$ is closed convex, so that the projection $P_{F(T)}$ is well defined. 
Lemma 8. Zhou (2008) Let $H$ be a Hilbert space, $C$ be a closed and convex subset of $H$, and $T: C \rightarrow H$ be a $k$-strict pseudo-contraction mapping. Define a mapping $V: C \rightarrow$ $H$ by $V x=\lambda x+(1-\lambda)$ Tx for all $x \in C$. Then, as $\lambda \in[k, 1)$, $V$ is a nonexpansive mapping such that $F(V)=F(T)$.

Lemma 9. Gu et al. (2011) Let $H$ be a Hilbert space and $C$ be a nonempty closed and convex subset of $H$. Let $T$ be a nonexpansive mapping of $C$ into itself such that $F(T) \neq \emptyset$. Then $\|T x-x\|^{2} \leq 2\left\langle x-T x, x-x^{\prime}\right\rangle, \quad \forall x^{\prime} \in F(T), \forall x \in C$.

\section{Main results}

Let us consider the net iterative scheme as follows:

$$
\left\{\begin{array}{l}
y_{n}=P_{C}\left[\beta_{n} S x_{n}+\left(1-\beta_{n}\right) x_{n}\right], \\
x_{n+1}=P_{C}\left[\alpha_{n} f\left(x_{n}\right)+\sum_{i=1}^{n}\left(\alpha_{i-1}-\alpha_{i}\right) V_{i} y_{n}\right], \quad \forall n \geq 1,
\end{array}\right.
$$

where $V_{i}=k_{i} I+\left(1-k_{i}\right) T_{i}, f: C \rightarrow H$ is a $\rho$ contraction mapping, $S: C \rightarrow H$ is a nonexpansive mapping, $\left\{T_{i}\right\}_{i=1}^{\infty}: C \rightarrow C$ is a countable family of $k_{i}$ strict pseudo-contraction mappings and $\cap_{i=1}^{\infty} F\left(T_{i}\right) \neq \emptyset$. Set $\alpha_{0}=1,\left\{\alpha_{n}\right\} \subset(0,1)$ is a strictly decreasing sequence and $\left\{\beta_{n}\right\} \subset(0,1)$. As we will see the convergence of the scheme depends on the choice of the parameters $\left\{\alpha_{n}\right\}$ and $\left\{\beta_{n}\right\}$. We list some possible hypotheses on them:

$$
\begin{aligned}
& \text { (H1) there exists } \gamma>0 \text { such that } \beta_{n} \leq \gamma \alpha_{n} ; \\
& \text { (H2) } \quad \lim _{n \rightarrow \infty} \beta_{n} / \alpha_{n}=\tau \in[0, \infty) ; \\
& \text { (H3) } \lim _{n \rightarrow \infty} \alpha_{n}=0 \text { and } \sum_{n=1}^{\infty} \alpha_{n}=\infty ; \\
& \text { (H4) } \sum_{n=1}^{\infty}\left|\alpha_{n}-\alpha_{n-1}\right|<\infty ; \\
& \text { (H5) } \sum_{n=1}^{\infty}\left|\beta_{n}-\beta_{n-1}\right|<\infty ; \\
& \text { (H6) } \quad \lim _{n \rightarrow \infty}\left|\alpha_{n}-\alpha_{n-1}\right| / \alpha_{n}=0 ; \\
& \text { (H7) } \lim _{n \rightarrow \infty}\left|\beta_{n}-\beta_{n-1}\right| / \beta_{n}=0 ; \\
& \text { (H8) } \quad \lim _{n \rightarrow \infty}\left[\left|\alpha_{n}-\alpha_{n-1}\right|+\left|\beta_{n}-\beta_{n-1}\right|\right] / \alpha_{n} \beta_{n}=0 ; \\
& \text { (H9) there exists a constant } K>0 \text { such that } \\
& \\
& \frac{1}{\alpha_{n}}\left|\frac{1}{\beta_{n}}-\frac{1}{\beta_{n i 1}}\right| \leq K .
\end{aligned}
$$

Proposition 1. Assume that (H1) holds. Then $\left\{x_{n}\right\}$ and $\left\{y_{n}\right\}$ are bounded.

Proof. Let $z \in \cap_{i=1}^{\infty} F\left(T_{i}\right)=\cap_{i=1}^{\infty} F\left(V_{i}\right)$. Then we have

$$
\begin{aligned}
\left\|x_{n+1}-z\right\| & =\left\|P_{C}\left[\alpha_{n} f\left(x_{n}\right)+\sum_{i=1}^{n}\left(\alpha_{i-1}-\alpha_{i}\right) V_{i} y_{n}\right]-P_{C}[z]\right\| \\
& \leq\left\|\alpha_{n} f\left(x_{n}\right)+\sum_{i=1}^{n}\left(\alpha_{i-1}-\alpha_{i}\right) V_{i} y_{n}-z\right\| \\
& =\left\|\alpha_{n}\left(f\left(x_{n}\right)-z\right)+\sum_{i=1}^{n}\left(\alpha_{i-1}-\alpha_{i}\right)\left(V_{i} y_{n}-z\right)\right\|
\end{aligned}
$$

$$
\begin{aligned}
& \leq \alpha_{n}\left\|f\left(x_{n}\right)-f(z)\right\|+\alpha_{n}\|f(z)-z\| \\
& +\sum_{i=1}^{n}\left(\alpha_{i-1}-\alpha_{i}\right)\left\|V_{i} y_{n}-z\right\| \\
& \leq \alpha_{n} \rho\left\|x_{n}-z\right\|+\alpha_{n}\|f(z)-z\| \\
& +\sum_{i=1}^{n}\left(\alpha_{i-1}-\alpha_{i}\right)\left\|y_{n}-z\right\| \\
& \leq \alpha_{n} \rho\left\|x_{n}-z\right\|+\alpha_{n}\|f(z)-z\| \\
& +\sum_{i=1}^{n}\left(\alpha_{i-1}-\alpha_{i}\right)\left\|\beta_{n} S x_{n}+\left(1-\beta_{n}\right) x_{n}-z\right\| \\
& \left.\leq \alpha_{n} \rho\left\|x_{n}-z\right\|+\alpha_{n}\|f(z)-z\|+\left(1-\beta_{n}\right)\left\|x_{n}-z\right\|\right) \\
& +\sum_{i=1}^{n}\left(\alpha_{i-1}-\alpha_{i}\right)\left(\beta_{n}\left\|S x_{n}-S z\right\|+\beta_{n}\|S z-z\|\right. \\
& \left.\leq \alpha_{n} \rho\left\|x_{n}-z\right\|+\alpha_{n}\|f(z)-z\|+\left(1-\beta_{n}\right)\left\|x_{n}-z\right\|\right) \\
& +\sum_{i=1}^{n}\left(\alpha_{i-1}-\alpha_{i}\right)\left(\beta_{n}\left\|x_{n}-z\right\|+\beta_{n}\|S z-z\|\right. \\
& =\alpha_{n} \rho\left\|x_{n}-z\right\|+\alpha_{n}\|f(z)-z\| \\
& +\sum_{i=1}^{n}\left(\alpha_{i-1}-\alpha_{i}\right)\left(\left\|x_{n}-z\right\|+\beta_{n}\|S z-z\|\right) \\
& =\alpha_{n} \rho\left\|x_{n}-z\right\|+\alpha_{n}\|f(z)-z\| \\
& +\left(1-\alpha_{n}\right)\left(\left\|x_{n}-z\right\|+\beta_{n}\|S z-z\|\right) \\
& =\left(1-\alpha_{n}(1-\rho)\right)\left\|x_{n}-z\right\|+\alpha_{n}\|f(z)-z\| \\
& +\left(1-\alpha_{n}\right) \beta_{n}\|S z-z\| \\
& \leq\left(1-\alpha_{n}(1-\rho)\right)\left\|x_{n}-z\right\|+\alpha_{n}\|f(z)-z\| \\
& +\beta_{n}\|S z-z\| \\
& \leq\left(1-\alpha_{n}(1-\rho)\right)\left\|x_{n}-z\right\|+\alpha_{n}[\|f(z)-z\| \\
& +\gamma\|S z-z\|] .
\end{aligned}
$$

So, by induction, one can obtain that

$\left\|x_{n}-z\right\| \leq \max \left\{\left\|x_{0}-z\right\|, \frac{1}{1-\rho}[\|f(z)-z\|+\gamma\|S z-z\|\}\right.$.

Hence $\left\{x_{n}\right\}$ is bounded. Of course $\left\{y_{n}\right\}$ is bounded too.

Proposition 2. Suppose that (H1) and (H3) hold. Also, assume that either (H4) and (H5) hold, or (H6) and (H7) hold. Then 
(1) $\left\{x_{n}\right\}$ is asymptotically regular, that is,

$$
\lim _{n \rightarrow \infty}\left\|x_{n+1}-x_{n}\right\|=0
$$

(2) the weak cluster points set $\omega_{w}\left(x_{n}\right) \subset \cap_{i=1}^{\infty} F\left(T_{i}\right)$.

Proof. Set $u_{n}=\alpha_{n} f\left(x_{n}\right)+\sum_{i=1}^{n}\left(\alpha_{i-1}-\alpha_{i}\right) V_{i} y_{n}$. From (16) and since $P_{C}$ is a nonexpansive mapping, we have

$$
\begin{aligned}
\left\|x_{n+1}-x_{n}\right\|= & \left\|P_{C}\left[u_{n}\right]-P_{C}\left[u_{n-1}\right]\right\| \\
\leq & \left\|u_{n}-u_{n-1}\right\| \\
= & \| \alpha_{n}\left(f\left(x_{n}\right)-f\left(x_{n-1}\right)\right)+\left(\alpha_{n}-\alpha_{n-1}\right) f\left(x_{n-1}\right) \\
& +\sum_{i=1}^{n}\left(\alpha_{i-1}-\alpha_{i}\right)\left(V_{i} y_{n}-V_{i} y_{n-1}\right) \\
& +\left(\alpha_{n-1}-\alpha_{n}\right) V_{n} y_{n-1}\left\|\leq \alpha_{n}\right\| f\left(x_{n}\right)-f\left(x_{n-1}\right) \| \\
& +\sum_{i=1}^{n}\left(\alpha_{i-1}-\alpha_{i}\right)\left\|y_{n}-y_{n-1}\right\| \\
& +\left|\alpha_{n}-\alpha_{n-1}\right|\left(\left\|f\left(x_{n-1}\right)\right\|+\left\|V_{n} y_{n-1}\right\|\right) \\
\leq & \alpha_{n} \rho\left\|x_{n}-x_{n-1}\right\|+\left(1-\alpha_{n}\right)\left\|y_{n}-y_{n-1}\right\| \\
& +\left|\alpha_{n}-\alpha_{n-1}\right|\left(\left\|f\left(x_{n-1}\right)\right\|+\left\|V_{n} y_{n-1}\right\|\right) .
\end{aligned}
$$

By definition of $y_{n}$ one obtain that

$$
\begin{aligned}
\left\|y_{n}-y_{n-1}\right\|= & \| P_{C}\left[\beta_{n} S x_{n}+\left(1-\beta_{n}\right) x_{n}\right] \\
& -P_{C}\left[\beta_{n-1} S x_{n-1}+\left(1-\beta_{n-1}\right) x_{n-1}\right] \| \\
\leq & \|\left(\beta_{n} S x_{n}+\left(1-\beta_{n}\right) x_{n}\right) \\
& -\left(\beta_{n-1} S x_{n-1}+\left(1-\beta_{n-1}\right) x_{n-1}\right) \| \\
= & \| \beta_{n}\left(S x_{n}-S x_{n-1}\right)+\left(\beta_{n}-\beta_{n-1}\right) S x_{n-1} \\
& +\left(1-\beta_{n-1}\right)\left(x_{n}-x_{n-1}\right)+\left(\beta_{n-1}-\beta_{n}\right) x_{n-1} \| \\
\leq & \left\|x_{n}-x_{n-1}\right\|+\left|\beta_{n}-\beta_{n-1}\right|\left(\left\|S x_{n-1}\right\|+\left\|x_{n-1}\right\|\right) .
\end{aligned}
$$

So, substituting (22) in (21), we obtain

$$
\begin{aligned}
\left\|x_{n+1}-x_{n}\right\| \leq & \alpha_{n} \rho\left\|x_{n}-x_{n-1}\right\|+\left(1-\alpha_{n}\right)\left[\left\|x_{n}-x_{n-1}\right\|\right. \\
& \left.+\left|\beta_{n}-\beta_{n-1}\right|\left(\left\|S x_{n-1}\right\|+\left\|x_{n-1}\right\|\right)\right] \\
& +\left|\alpha_{n}-\alpha_{n-1}\right|\left(\left\|f\left(x_{n-1}\right)\right\|+\left\|V_{n} y_{n-1}\right\|\right) \\
\leq & \left(1-(1-\rho) \alpha_{n}\right)\left\|x_{n}-x_{n-1}\right\| \\
& +\left|\beta_{n}-\beta_{n-1}\right|\left(\left\|S x_{n-1}\right\|+\left\|x_{n-1}\right\|\right) \\
& +\left|\alpha_{n}-\alpha_{n-1}\right|\left(\left\|f\left(x_{n-1}\right)\right\|+\left\|V_{n} y_{n-1}\right\|\right) .
\end{aligned}
$$

By Proposition 1, we say

$$
\begin{aligned}
M:=\max & \left\{\sup _{n \geq 1}\left\{\left\|S x_{n-1}\right\|+\left\|x_{n-1}\right\|\right\},\right. \\
& \left.\sup _{n \geq 1}\left\{\left\|f\left(x_{n-1}\right)\right\|+\left\|V_{n} y_{n-1}\right\|\right\}\right\} .
\end{aligned}
$$

So, we have

$$
\begin{aligned}
\left\|x_{n+1}-x_{n}\right\| \leq & \left(1-(1-\rho) \alpha_{n}\right)\left\|x_{n}-x_{n-1}\right\| \\
& +M\left[\left|\alpha_{n}-\alpha_{n-1}\right|+\left|\beta_{n}-\beta_{n-1}\right|\right]
\end{aligned}
$$

So, if (H4) and (H5) hold, we obtain the asymptotic regularity by Lemma 5, if instead, (H6) and (H7) hold, from (H1), we can write

$$
\begin{aligned}
\left\|x_{n+1}-x_{n}\right\| \leq & \left(1-(1-\rho) \alpha_{n}\right)\left\|x_{n}-x_{n-1}\right\| \\
& +M \alpha_{n}\left[\frac{\left|\alpha_{n}-\alpha_{n-1}\right|}{\alpha_{n}}+\frac{\left|\beta_{n}-\beta_{n-1}\right|}{\alpha_{n}}\right] \\
\leq & \left(1-(1-\rho) \alpha_{n}\right)\left\|x_{n}-x_{n-1}\right\| \\
& +M \alpha_{n}\left[\frac{\left|\alpha_{n}-\alpha_{n-1}\right|}{\alpha_{n}}+\gamma \frac{\left|\beta_{n}-\beta_{n-1}\right|}{\beta_{n}}\right] .
\end{aligned}
$$

By Lemma 5, we obtain the asymptotics regularity.

In order to prove (2), since $V_{i} x_{n} \in C$ for each $i \geq 1$ and $\sum_{n=1}^{\infty}\left(\alpha_{n-1}-\alpha_{n}\right)+\alpha_{n}=1$, we have

$$
\sum_{i=1}^{n}\left(\alpha_{i-1}-\alpha_{i}\right) V_{i} x_{n}+\alpha_{n} p \in C, \quad \forall p \in C
$$

Now, fixing $p \in \cap_{i=1}^{\infty} F\left(V_{i}\right)$, from (16), we have $\sum_{i=1}^{n}\left(\alpha_{i-1}-\alpha_{i}\right)\left(x_{n}-V_{i} x_{n}\right)$

$$
\begin{aligned}
= & P_{C}\left[u_{n}\right]+\left(1-\alpha_{n}\right) x_{n}-\left(\sum_{i=1}^{n}\left(\alpha_{i-1}-\alpha_{i}\right) V_{i} x_{n}+\alpha_{n} p\right) \\
& +\alpha_{n} p-x_{n+1} \\
= & P_{C}\left[u_{n}\right]-P_{C}\left[\sum_{i=1}^{n}\left(\alpha_{i-1}-\alpha_{i}\right) V_{i} x_{n}+\alpha_{n} p\right] \\
& +\left(1-\alpha_{n}\right)\left(x_{n}-x_{n+1}\right)+\alpha_{n}\left(p-x_{n+1}\right) .
\end{aligned}
$$


It follows that $\sum_{i=1}^{n}\left(\alpha_{i-1}-\alpha_{i}\right)\left\langle x_{n}-V_{i} x_{n}, x_{n}-z\right\rangle$

$$
\begin{aligned}
= & \left\langle P_{C}\left[u_{n}\right]-P_{C}\left[\sum_{i=1}^{n}\left(\alpha_{i-1}-\alpha_{i}\right) V_{i} x_{n}+\alpha_{n} p\right], x_{n}-z\right\rangle \\
& +\left(1-\alpha_{n}\right)\left\langle x_{n}-x_{n+1}, x_{n}-z\right\rangle+\alpha_{n}\left\langle p-x_{n+1}, x_{n}-z\right\rangle \\
\leq & \left\|u_{n}-\sum_{i=1}^{n}\left(\alpha_{i-1}-\alpha_{i}\right) V_{i} x_{n}+\alpha_{n} p\right\|\left\|x_{n}-z\right\| \\
& +\left(1-\alpha_{n}\right)\left\|x_{n}-x_{n+1}\right\|\left\|x_{n}-z\right\|+\alpha_{n}\left\|p-x_{n+1}\right\|\left\|x_{n}-z\right\| \\
= & \left\|\alpha_{n}\left(f\left(x_{n}\right)-p\right)+\sum_{i=1}^{n}\left(\alpha_{i-1}-\alpha_{i}\right)\left(V_{i} y_{n}-V_{i} x_{n}\right)\right\|\left\|x_{n}-z\right\| \\
& +\left(1-\alpha_{n}\right)\left\|x_{n}-x_{n+1}\right\|\left\|x_{n}-z\right\|+\alpha_{n}\left\|p-x_{n+1}\right\|\left\|x_{n}-z\right\| \\
& +\left(1-\alpha_{n}\right)\left\|x_{n}-x_{n+1}\right\|\left\|x_{n}-z\right\|+\alpha_{n}\left\|p-x_{n+1}\right\|\left\|x_{n}-z\right\| \\
\leq & \alpha_{n}\left\|f\left(x_{n}\right)-p\right\|\left\|x_{n}-z\right\|+\sum_{i=1}^{n}\left(\alpha_{i-1}-\alpha_{i}\right)\left\|y_{n}-x_{n}\right\|\left\|x_{n}-z\right\| \\
& +\left(1-\alpha_{n}\right)\left\|x_{n}-x_{n+1}\right\|\left\|x_{n}-z\right\|+\alpha_{n}\left\|p-x_{n+1}\right\|\left\|x_{n}-z\right\| \\
& \alpha_{n}\left\|f\left(x_{n}\right)-p\right\|\left\|x_{n}-z\right\|+\sum_{i=1}^{n}\left(\alpha_{i-1}-\alpha_{i}\right) \beta_{n}\left\|S x_{n}-x_{n}\right\|\left\|x_{n}-z\right\| \\
& +x_{n+1}\|\| x_{n}-z\left\|+\alpha_{n}\right\| p-x_{n+1}\|\| x_{n}-z \| .
\end{aligned}
$$

Now, from Lemma 9 and (27), we get $\frac{1}{2} \sum_{i=1}^{n}\left(\alpha_{i-1}-\right.$ $\left.\alpha_{i}\right)\left\|x_{n}-V_{i} x_{n}\right\|^{2}$

$$
\begin{aligned}
\leq & \sum_{i=1}^{n}\left(\alpha_{i-1}-\alpha_{i}\right)\left\langle x_{n}-V_{i} x_{n}, x_{n}-z\right\rangle \\
\leq & \alpha_{n}\left\|f\left(x_{n}\right)-p\right\|\left\|x_{n}-z\right\|+\left(1-\alpha_{n}\right) \beta_{n}\left\|S x_{n}-x_{n}\right\|\left\|x_{n}-z\right\| \\
& +\left(1-\alpha_{n}\right)\left\|x_{n}-x_{n+1}\right\|\left\|x_{n}-z\right\|+\alpha_{n}\left\|p-x_{n+1}\right\|\left\|x_{n}-z\right\| .
\end{aligned}
$$

By (H1) and (H3), it follows that $\beta_{n} \rightarrow 0$, as $n \rightarrow \infty$, so that

$$
\lim _{n \rightarrow \infty} \sum_{i=1}^{n}\left(\alpha_{i-1}-\alpha_{i}\right)\left\|x_{n}-V_{i} x_{n}\right\|^{2}=0 .
$$

Since $\left(\alpha_{i-1}-\alpha_{i}\right)\left\|x_{n}-V_{i} x_{n}\right\|^{2} \leq \sum_{i=1}^{n}\left(\alpha_{i-1}-\alpha_{i}\right) \| x_{n}-$ $V_{i} x_{n} \|^{2}$ for each $i \geq 1$ and $\left\{\alpha_{n}\right\}$ is strictly decreasing, one has

$$
\lim _{n \rightarrow \infty}\left\|x_{n}-V_{i} x_{n}\right\|=0, \quad \forall i \geq 1 .
$$

Hence, we obtain

$$
\lim _{n \rightarrow \infty}\left\|x_{n}-T_{i} x_{n}\right\|=\lim _{n \rightarrow \infty} \frac{\left\|x_{n}-V_{i} x_{n}\right\|}{\left(1-k_{i}\right)}=0, \quad \forall i \geq 1 .
$$

Since $\left\{x_{n}\right\}$ is asymptotically regular and demiclosedness principle, we obtain the proposition.

Corollary 1. Suppose that the hypotheses of Proposition 2 hold. Then

(i) $\lim _{n \rightarrow \infty}\left\|x_{n}-y_{n}\right\|=0$;

(ii) $\lim _{n \rightarrow \infty}\left\|x_{n}-V_{i} y_{n}\right\|=0, \quad \forall i \geq 1$;

(iii) $\lim _{n \rightarrow \infty}\left\|y_{n}-V_{i} y_{n}\right\|=0, \quad \forall i \geq 1$.

Proof. To prove $(i)$, we can observe that

$$
\left\|x_{n}-y_{n}\right\| \leq \beta_{n}\left\|x_{n}-S x_{n}\right\| \text {. }
$$

Since $\beta_{n} \rightarrow 0$ as $n \rightarrow \infty$, we obtain $(i)$.

To prove $(i i)$, we observe that

$$
\left\|y_{n}-V_{i} x_{n}\right\| \leq\left\|y_{n}-x_{n}\right\|+\left\|x_{n}-V_{i} x_{n}\right\|, \quad \forall i \geq 1
$$

and

$$
\left\|x_{n}-V_{i} y_{n}\right\| \leq\left\|x_{n}-y_{n}\right\|+\left\|y_{n}-V_{i} x_{n}\right\|, \quad \forall i \geq 1 .
$$

Since $\left\|y_{n}-x_{n}\right\| \rightarrow 0$ and $\left\|x_{n}-V_{i} x_{n}\right\| \rightarrow 0$ as $n \rightarrow \infty$, $\forall i \geq 1$, then $\left\|y_{n}-V_{i} x_{n}\right\| \rightarrow 0$, that is, we obtain (ii). To prove (iii), we can observe that

$$
\left\|y_{n}-V_{i} y_{n}\right\| \leq\left\|x_{n}-y_{n}\right\|+\left\|x_{n}-V_{i} y_{n}\right\|, \quad \forall i \geq 1 .
$$

By $(i)$ and (ii), we obtain (iii).

Theorem 1. Let $C$ be a nonempty closed and convex subset of a real Hilbert space $H$. Let $f: C \rightarrow H$ be a $\rho$-contraction mapping, $S: C \rightarrow H$ be a nonexpansive mapping and $\left\{T_{i}\right\}_{i=1}^{\infty}: C \rightarrow C$ be a countable family of $k_{i^{-}}$ strict pseudo-contraction mappings and $\mathcal{F}=\cap_{i=1}^{\infty} F\left(T_{i}\right) \neq$ $\emptyset$. Let $\alpha_{0}=1$, and $x_{1} \in C$ and define the sequence $\left\{x_{n}\right\}$ by

$$
\left\{\begin{array}{l}
y_{n}=P_{C}\left[\beta_{n} S x_{n}+\left(1-\beta_{n}\right) x_{n}\right], \\
x_{n+1}=P_{C}\left[\alpha_{n} f\left(x_{n}\right)+\sum_{i=1}^{n}\left(\alpha_{i-1}-\alpha_{i}\right) V_{i} y_{n}\right], \quad \forall n \geq 1,
\end{array}\right.
$$

where $\left\{\alpha_{n}\right\} \subset(0,1)$ and $\left\{\alpha_{n}\right\}$ is a strictly decreasing sequence, $V_{i}=k_{i} I+\left(1-k_{i}\right) T_{i},\left\{\beta_{n}\right\} \subset(0,1)$ and $\left\{\alpha_{n}\right\}$ and $\left\{\beta_{n}\right\}$ are sequences satisfying the conditions (H2) with $\tau=0$, (H3), either (H4) and (H5), or (H6) and (H7). Then the sequence $\left\{x_{n}\right\}$ converges strongly to a point $z \in \mathcal{F}$, which is the unique solution of the variational inequality:

$$
\langle(I-f) z, x-z\rangle \geq 0, \quad \forall x \in \mathcal{F} .
$$


Proof. First of all, since $P_{\mathcal{F}} f$ is a contraction. By Banach contraction principle, so there exists a unique $z \in \mathcal{F}$ such that $z=P_{\mathcal{F}} f(z)$, Moreover, from Lemma 3(1), we have

$$
\langle f(z)-z, y-z\rangle \leq 0, \quad \forall y \in \mathcal{F} .
$$

Since (H2) implies (H1), thus $\left\{x_{n}\right\}$ is bounded. Moreover, since either (H4) and (H5) or (H6) and (H7) then $\left\{x_{n}\right\}$ is asymptotically regular. Similarly, by Proposition 2 , the weak cluster points set of $x_{n}$, that is, $\omega_{w}\left(x_{n}\right)$, is a subset of $\mathcal{F}$.

Let $\left\{x_{n_{k}}\right\}$ be a subsequence of $\left\{x_{n}\right\}$ such that

$$
\limsup _{n \rightarrow \infty}\left\langle f(z)-z, x_{n}-z\right\rangle=\lim _{k \rightarrow \infty}\left\langle f(z)-z, x_{n_{k}}-z\right\rangle,
$$

and $x_{n_{k}} \rightarrow x$. So, it follows that $x^{\prime} \in \mathcal{F}$. Then, we also have

$$
\lim _{k \rightarrow \infty}\left\langle f(z)-z, x_{n_{k}}-z\right\rangle=\left\langle f(z)-z, x^{\prime}-z\right\rangle \leq 0 .
$$

Set $u_{n}=\alpha_{n} f\left(x_{n}\right)+\sum_{i=1}^{n}\left(\alpha_{i-1}-\alpha_{i}\right) V_{i} y_{n}$, we obtain

$\left\|x_{n+1}-z\right\|^{2}=\left\langle P_{C}\left[u_{n}\right]-u_{n}, P_{C}\left[u_{n}\right]-z\right\rangle+\left\langle u_{n}-z, x_{n+1}-z\right\rangle$.

By Lemma 3(1), we have

$$
\left\langle P_{C}\left[u_{n}\right]-u_{n}, P_{C}\left[u_{n}\right]-z\right\rangle \leq 0 .
$$

From (32) and (33), it follows that $\left\|x_{n+1}-z\right\|^{2}$

$$
\begin{aligned}
& \leq\left\langle u_{n}-z, x_{n+1}-z\right\rangle=\alpha_{n}\left\langle f\left(x_{n}\right)-f(z), x_{n+1}-z\right\rangle \\
& \quad+\alpha_{n}\left\langle f(z)-z, x_{n+1}-z\right\rangle \sum_{i=1}^{n}\left(\alpha_{i-1}-\alpha_{i}\right) \\
& \quad \times\left\langle V_{i} y_{n}-z, x_{n+1}-z\right\rangle \\
& \leq \alpha_{n} \rho\left\|x_{n}-z\right\|\left\|x_{n+1}-z\right\|+\alpha_{n}\left\langle f(z)-z, x_{n+1}-z\right\rangle \\
& \quad \times\left(1-\alpha_{n}\right)\left\|y_{n}-z\right\|\left\|x_{n+1}-z\right\| \leq \alpha_{n} \rho\left\|x_{n}-z\right\| \\
& \quad \times\left\|x_{n+1}-z\right\|+\alpha_{n}\left\langle f(z)-z, x_{n+1}-z\right\rangle \\
& \quad \times\left(1-\alpha_{n}\right)\left\|\beta_{n} S x_{n}+\left(1-\beta_{n}\right) x_{n}-z\right\|\left\|x_{n+1}-z\right\|
\end{aligned}
$$

$$
\begin{aligned}
& \leq \alpha_{n} \rho\left\|x_{n}-z\right\|\left\|x_{n+1}-z\right\|+\alpha_{n}\left\langle f(z)-z, x_{n+1}-z\right\rangle \\
& \times\left(1-\alpha_{n}\right)\left\|x_{n}-z\right\|\left\|x_{n+1}-z\right\| \\
& +\left(1-\alpha_{n}\right) \beta_{n}\|S z-z\|\left\|x_{n+1}-z\right\| \\
& =\left[1-\alpha_{n}(1-\rho)\right]\left\|x_{n}-z\right\|\left\|x_{n+1}-z\right\| \\
& +\alpha_{n}\left\langle f(z)-z, x_{n+1}-z\right\rangle \\
& +\left(1-\alpha_{n}\right) \beta_{n}\|S z-z\|\left\|x_{n+1}-z\right\| \\
& \leq\left[\frac{1-\alpha_{n}(1-\rho)}{2}\right]\left[\left\|x_{n}-z\right\|^{2}+\left\|x_{n+1}-z\right\|^{2}\right] \\
& +\alpha_{n}\left\langle f(z)-z, x_{n+1}-z\right\rangle+\left(1-\alpha_{n}\right) \beta_{n}\|S z-z\| \\
& \times\left\|x_{n+1}-z\right\| \leq\left[1-\frac{2(1-\rho) \alpha_{n}}{1+(1-\rho) \alpha_{n}}\right]\left\|x_{n}-z\right\|^{2} \\
& +\left[\frac{2 \alpha_{n}}{1+(1-\rho) \alpha_{n}}\right] \cdot\left\langle f(z)-z, x_{n+1}-z\right\rangle \\
& +\left[\frac{2\left(1-\alpha_{n}\right) \beta_{n}}{1+(1-\rho) \alpha_{n}}\right]\|S z-z\|\left\|x_{n+1}-z\right\| \\
& =\left[1-\frac{2(1-\rho) \alpha_{n}}{1+(1-\rho) \alpha_{n}}\right]\left\|x_{n}-z\right\|^{2}+\left[\frac{2(1-\rho) \alpha_{n}}{1+(1-\rho) \alpha_{n}}\right] \\
& \times\left\{\frac{1}{1-\rho}\left\langle f(z)-z, x_{n+1}-z\right\rangle+\frac{\left(1-\alpha_{n}\right) \beta_{n}}{(1-\rho) \alpha_{n}}\right. \\
& \left.\times\|S z-z\|\left\|x_{n+1}-z\right\|\right\} .
\end{aligned}
$$

Let $\gamma_{n}=\frac{2(1-\rho) \alpha_{n}}{1+(1-\rho) \alpha_{n}}$ and $\delta_{n}=\frac{2(1-\rho) \alpha_{n}}{1+(1-\rho) \alpha_{n}}\left\{\frac{1}{1-\rho}\langle f(z)-z\right.$, $\left.\left.x_{n+1}-z\right\rangle+\frac{\left(1-\alpha_{n}\right) \beta_{n}}{(1-\rho) \alpha_{n}} \cdot\|S z-z\|\left\|x_{n+1}-z\right\|\right\}$ for all $n \geq 1$. Since

$$
\begin{aligned}
& \limsup _{n \rightarrow \infty}\left\{\frac{1}{1-\rho}\left\langle f(z)-z, x_{n+1}-z\right\rangle+\frac{\left(1-\alpha_{n}\right) \beta_{n}}{(1-\rho) \alpha_{n}}\right. \\
&\left.\times\|S z-z\|\left\|x_{n+1}-z\right\|\right\} \leq 0, \\
& \sum_{i=1}^{\infty} \alpha_{n}=\infty \text { and } \frac{2(1-\rho) \alpha_{n}}{1+(1-\rho) \alpha_{n}} \geq(1-\rho) \alpha_{n}, \text { we have } \\
& \sum_{n=1}^{\infty} \gamma_{n}=\infty \text { and } \limsup _{n \rightarrow \infty} \frac{\delta_{n}}{\gamma_{n}} \leq 0 .
\end{aligned}
$$

Hence, by Lemma 5 , we conclude that $x_{n} \rightarrow z$ as $n \rightarrow \infty$. 
Remark 1. In the iterative scheme (30), if we set $f \equiv 0$, then we get $x_{n} \rightarrow z=P_{\mathcal{F}} 0$. In this case, from (31), it follows that

$$
\langle z, z-x\rangle \leq 0, \quad \forall x \in \mathcal{F}
$$

That is

$$
\|z\|^{2} \leq\langle z, x\rangle \leq\|z\|\|x\|, \quad \forall x \in \mathcal{F} .
$$

Therefore, the point $z$ is the unique solution to the following quadratic minimization problem:

$$
z=\arg \min _{x \in \mathcal{F}}\|x\|^{2}
$$

By changing the restrictions on parameters in Theorem 1, we obtain the following results.

Theorem 2. Let $C$ be a nonempty closed and convex subset of a real Hilbert space $H$. Let $f: C \rightarrow H$ be a $\rho$ contraction mapping, $S: C \rightarrow C$ be a nonexpansive mapping and $\left\{T_{i}\right\}_{i=1}^{\infty}: C \rightarrow C$ be a countable family of $k_{i}$-strict pseudo-contraction mappings and $\mathcal{F}=\cap_{i=1}^{\infty} F\left(T_{i}\right) \neq$ $\emptyset$. Let $\alpha_{0}=1$, and $x_{1} \in C$ and define the sequence $\left\{x_{n}\right\}$ by

$$
\left\{\begin{array}{l}
y_{n}=P_{C}\left[\beta_{n} S x_{n}+\left(1-\beta_{n}\right) x_{n}\right]=\beta_{n} S x_{n}+\left(1-\beta_{n}\right) x_{n} \\
x_{n+1}=P_{C}\left[\alpha_{n} f\left(x_{n}\right)+\sum_{i=1}^{n}\left(\alpha_{i-1}-\alpha_{i}\right) V_{i} y_{n}\right], \quad \forall n \geq 1
\end{array}\right.
$$

where $\left\{\alpha_{n}\right\} \subset(0,1)$ and $\left\{\alpha_{n}\right\}$ is a strictly decreasing sequence, $V_{i}=k_{i} I+\left(1-k_{i}\right) T_{i},\left\{\beta_{n}\right\} \subset(0,1)$ and $\left\{\alpha_{n}\right\}$ and $\left\{\beta_{n}\right\}$ are sequences satisfying the conditions (H2) with $\tau \in(0, \infty)$, (H3), (H8) and (H9). Then the sequence $\left\{x_{n}\right\}$ converges strongly to a point $x^{*} \in \mathcal{F}$, which is the unique solution of the variational inequality:

$$
\left\langle\frac{1}{\tau}(I-f) x^{*}+(I-S) x^{*}, x-x^{*}\right\rangle \geq 0, \quad \forall x \in \mathcal{F} .
$$

Proof. First, we shows that (49) has the unique solution. Let $x^{\prime}$ and $x^{*}$ be two solutions. Then, since $x^{\prime}$ is solution, for $y=x^{*}$ one has

$$
\left\langle(I-f) x^{\prime}, x^{\prime}-x^{*}\right\rangle \leq \tau\left\langle(I-S) x^{\prime}, x^{*}-x^{\prime}\right\rangle
$$

and

$$
\left\langle(I-f) x^{*}, x^{*}-x^{\prime}\right\rangle \leq \tau\left\langle(I-S) x^{*}, x^{\prime}-x^{*}\right\rangle .
$$

Adding (36) and (37), we obtain

$$
\begin{aligned}
(1-\rho)\left\|x^{\prime}-x^{*}\right\|^{2} & \leq\left\langle(I-f) x^{\prime}-(I-f) x^{*}, x^{\prime}-x^{*}\right\rangle \\
& \leq-\rho\left\langle(I-S) x^{\prime}-(I-S) x^{*}, x^{\prime}-x^{*}\right\rangle \leq 0
\end{aligned}
$$

so $x^{\prime}=x^{*}$. Also now the condition (H2) with $0<\tau<\infty$ implies (H1) so the sequence $\left\{x_{n}\right\}$ is bounded. Moreover, since (H8) implies (H6) and (H7), then $\left\{x_{n}\right\}$ is asymptotically regular. Similarly, by Proposition 2 , the weak cluster points set of $x_{n}$, i.e., $\omega_{w}\left(x_{n}\right)$, is a subset of $\mathcal{F}$.

From (20)-(24), we observe that

$$
\begin{aligned}
& \left.\frac{\left\|x_{n+1}-x_{n}\right\|}{\beta_{n}} \leq \frac{\left\|u_{n}-u_{n-1}\right\|}{\beta_{n}} \leq[1-(1-\rho)) \alpha_{n}\right] \frac{\left\|x_{n}-x_{n-1}\right\|}{\beta_{n}} \\
& +M\left[\frac{\left|\alpha_{n}-\alpha_{n-1}\right|}{\beta_{n}}+\frac{\left|\beta_{n}-\beta_{n-1}\right|}{\beta_{n}}\right] \\
& \left.=[1-(1-\rho)) \alpha_{n}\right] \frac{\left\|x_{n}-x_{n-1}\right\|}{\beta_{n-1}} \\
& \left.+[1-(1-\rho)) \alpha_{n}\right]\left\|x_{n}-x_{n-1}\right\|\left[\frac{1}{\beta_{n}} v-\frac{1}{\beta_{n-1}}\right] \\
& +M\left[\frac{\left|\alpha_{n}-\alpha_{n-1}\right|}{\beta_{n}}+\frac{\left|\beta_{n}-\beta_{n-1}\right|}{\beta_{n}}\right] \\
& \left.\leq[1-(1-\rho)) \alpha_{n}\right] \frac{\left\|x_{n}-x_{n-1}\right\|}{\beta_{n-1}} \\
& +\left\|x_{n}-x_{n-1}\right\|\left[\frac{1}{\beta_{n}}-\frac{1}{\beta_{n-1}}\right] \\
& +M\left[\frac{\left|\alpha_{n}-\alpha_{n-1}\right|}{\beta_{n}}+\frac{\left|\beta_{n}-\beta_{n-1}\right|}{\beta_{n}}\right] \\
& \left.\leq[1-(1-\rho)) \alpha_{n}\right] \frac{\left\|x_{n}-x_{n-1}\right\|}{\beta_{n-1}}+\alpha_{n} K\left\|x_{n}-x_{n-1}\right\| \\
& +M\left[\frac{\left|\alpha_{n}-\alpha_{n-1}\right|}{\beta_{n}}+\frac{\left|\beta_{n}-\beta_{n-1}\right|}{\beta_{n}}\right] \\
& \left.\leq[1-(1-\rho)) \alpha_{n}\right] \frac{\left\|u_{n}-u_{n-1}\right\|}{\beta_{n-1}}+\alpha_{n} K\left\|x_{n}-x_{n-1}\right\|
\end{aligned}
$$

$$
+M\left[\frac{\left|\alpha_{n}-\alpha_{n-1}\right|}{\beta_{n}}+\frac{\left|\beta_{n}-\beta_{n-1}\right|}{\beta_{n}}\right] .
$$


Let $\gamma_{n}=(1-\rho) \alpha_{n}$ and $\delta_{n}=\alpha_{n} K\left\|x_{n}-x_{n-1}\right\|+$ $M\left[\frac{\left|\alpha_{n}-\alpha_{n-1}\right|}{\beta_{n}}+\frac{\left|\beta_{n}-\beta_{n-1}\right|}{\beta_{n}}\right]$. From condition (H3) and (H8), we have

$$
\sum_{i=1}^{\infty} \gamma_{n}=\infty \quad \text { and } \quad \lim _{n \rightarrow \infty} \frac{\delta_{n}}{\gamma_{n}}=0
$$

By Lemma 5, we obtain

$$
\begin{aligned}
& \lim _{n \rightarrow \infty} \frac{\left\|x_{n+1}-x_{n}\right\|}{\beta_{n}}=0, \\
& \lim _{n \rightarrow \infty} \frac{\left\|u_{n+1}-u_{n}\right\|}{\beta_{n}}=\lim _{n \rightarrow \infty} \frac{\left\|u_{n+1}-u_{n}\right\|}{\alpha_{n}}=0 .
\end{aligned}
$$

From (34), we have

$$
\begin{aligned}
x_{n}-x_{n-1}= & \left(1-\alpha_{n}\right) x_{n}-\left[P_{C}\left[u_{n}\right]-u_{n}+\alpha_{n} f\left(x_{n}\right)\right. \\
& \left.+\sum_{i=1}^{n}\left(\alpha_{i-1}-\alpha_{i}\right)\left(V_{i} y_{n}-y_{n}\right)+\left(1-\alpha_{n}\right) y_{n}\right] \\
= & \left(1-\alpha_{n}\right) \beta_{n}\left(x_{n}-S x_{n}\right)+\left(u_{n}-P_{C}\left[u_{n}\right]\right) \\
& +\sum_{i=1}^{n}\left(\alpha_{i-1}-\alpha_{i}\right)\left(y_{n}-V_{i} y_{n}\right)+\alpha_{n}\left(x_{n}-f\left(x_{n}\right)\right) .
\end{aligned}
$$

It follows that

$$
\begin{aligned}
\frac{x_{n}-x_{n-1}}{\left(1-\alpha_{n}\right) \beta_{n}}= & \left(x_{n}-S x_{n}\right)+\frac{1}{\left(1-\alpha_{n}\right) \beta_{n}}\left(u_{n}-P_{C}\left[u_{n}\right]\right) \\
& +\frac{1}{\left(1-\alpha_{n}\right) \beta_{n}} \sum_{i=1}^{n}\left(\alpha_{i-1}-\alpha_{i}\right)\left(y_{n}-V_{i} y_{n}\right) \\
& +\frac{\alpha_{n}}{\left(1-\alpha_{n}\right) \beta_{n}}\left(x_{n}-f\left(x_{n}\right)\right) .
\end{aligned}
$$

Let $v_{n}=\frac{x_{n}-x_{n-1}}{\left(1-\alpha_{n}\right) \beta_{n}}$. For all $z \in \mathcal{F}=\cap_{i=1}^{\infty} F\left(T_{i}\right)=$ $\cap_{i=1}^{\infty} F\left(V_{i}\right)$, we get

$$
\begin{aligned}
\left\langle v_{n}, x_{n}-z\right\rangle= & \frac{1}{\left(1-\alpha_{n}\right) \beta_{n}}\left\langle u_{n}-P_{C}\left[u_{n}\right], P_{C}\left[u_{n-1}\right]-z\right\rangle \\
& +\frac{\alpha_{n}}{\left(1-\alpha_{n}\right) \beta_{n}}\left\langle(I-f) x_{n}, x_{n}-z\right\rangle \\
& +\left\langle x_{n}-S x_{n}, x_{n}-z\right\rangle+\frac{1}{\left(1-\alpha_{n}\right) \beta_{n}} \\
& \sum_{i=1}^{n}\left(\alpha_{i-1}-\alpha_{i}\right)\left\langle y_{n}-V_{i} y_{n}, x_{n}-z\right\rangle .
\end{aligned}
$$

By Lemma 4, we have

$$
\begin{aligned}
\left\langle x_{n}-S x_{n}, x_{n}-z\right\rangle= & \left\langle(I-S) x_{n}-(I-S) z, x_{n}-z\right\rangle \\
& +\left\langle(I-S) z, x_{n}-z\right\rangle \geq\left\langle(I-S) z, x_{n}-z\right\rangle,
\end{aligned}
$$

$$
\begin{aligned}
\left\langle(I-f) x_{n}, x_{n}-z\right\rangle= & \left\langle(I-f) x_{n}-(I-f) z, x_{n}-z\right\rangle \\
& +\left\langle(I-f) z, x_{n}-z\right\rangle \geq(1-\rho)\left\|x_{n}-z\right\|^{2} \\
& +\left\langle(I-f) z, x_{n}-z\right\rangle
\end{aligned}
$$

and

$$
\begin{aligned}
\left\langle y_{n}-V_{i} y_{n}, x_{n}-z\right\rangle= & \left\langle\left(I-V_{i}\right) y_{n}-\left(I-V_{i}\right) z, x_{n}-y_{n}\right\rangle \\
& +\left\langle\left(I-V_{i}\right) y_{n}-\left(I-V_{i}\right) z, y_{n}-z\right\rangle \\
\geq & \left\langle\left(I-V_{i}\right) y_{n}-\left(I-V_{i}\right) z, x_{n}-y_{n}\right\rangle \\
= & \beta_{n}\left\langle\left(I-V_{i}\right) y_{n}, x_{n}-S x_{n}\right\rangle, \quad \forall i \geq 1 .
\end{aligned}
$$

By Lemma 3(1), we obtain

$$
\begin{aligned}
\left\langle u_{n}-\right. & \left.P_{C}\left[u_{n}\right], P_{C}\left[u_{n-1}\right]-z\right\rangle \\
= & \left\langle u_{n}-P_{C}\left[u_{n}\right], P_{C}\left[u_{n-1}\right]-P_{C}\left[u_{n}\right]\right\rangle \\
& +\left\langle u_{n}-P_{C}\left[u_{n}\right], P_{C}\left[u_{n}\right]-z\right\rangle \\
\geq & \left\langle u_{n}-P_{C}\left[u_{n}\right], P_{C}\left[u_{n-1}\right]-P_{C}\left[u_{n}\right]\right\rangle
\end{aligned}
$$

Now, from (38)-(42), it follows that

$$
\begin{aligned}
\left\|x_{n}-z\right\|^{2} \leq & \frac{\left(1-\alpha_{n}\right) \beta_{n}}{(1-\rho) \alpha_{n}}\left[\left\langle v_{n}, x_{n}-z\right\rangle-\left\langle(I-S) z, x_{n}-z\right\rangle\right] \\
& +\frac{\left\|u_{n-1}-u_{n}\right\|}{(1-\rho) \alpha_{n}}\left\|u_{n}-P_{C}\left[u_{n}\right]\right\| \\
& -\frac{1}{1-\rho}\left\langle(I-f) z, x_{n}-z\right\rangle-\frac{\beta_{n}}{(1-\rho) \alpha_{n}} \\
& \sum_{i=1}^{n}\left(\alpha_{i-1}-\alpha_{i}\right)\left\langle\left(I-V_{i}\right) y_{n}, x_{n}-S x_{n}\right\rangle,
\end{aligned}
$$

since $v_{n} \rightarrow 0$ and $\left(I-V_{i}\right) y_{n} \rightarrow 0$, as $n \rightarrow \infty$, then every weak cluster point of $\left\{x_{n}\right\}$ is also a strong cluster point. By Proposition 2, $\left\{x_{n}\right\}$ is bounded, thus there exists 
a subsequence $\left\{x_{n_{k}}\right\}$ converging to $x^{*}$. For all $z \in \mathcal{F}$ by (38), we compute

$$
\begin{aligned}
\left\langle(I-f) x_{n_{k}}, x_{n_{k}}-z\right\rangle= & \frac{\left(1-\alpha_{n_{k}}\right) \beta_{n_{k}}}{\alpha_{n_{k}}}\left\langle v_{n_{k}}, x_{n_{k}}-z\right\rangle \\
& -\frac{1}{\alpha_{n_{k}}}\left\langle u_{n_{k}}-P_{C}\left[u_{n_{k}}\right], P_{C}\left[u_{n_{k}-1}\right]-z\right\rangle \\
& -\frac{\left(1-\alpha_{n_{k}}\right) \beta_{n_{k}}}{\alpha_{n_{k}}}\left\langle x_{n_{k}}-S x_{n_{k}}, x_{n_{k}}-z\right\rangle \\
& -\frac{1}{\left(\alpha_{n_{k}}\right.} \sum_{i=1}^{n_{k}}\left(\alpha_{i-1}-\alpha_{i}\right) \\
& \times\left\langle y_{n_{k}}-V_{i} y_{n_{k}}, x_{n_{k}}-z\right\rangle \\
\leq & \frac{\left(1-\alpha_{n_{k}}\right) \beta_{n_{k}}}{\alpha_{n_{k}}}\left\langle v_{n_{k}}, x_{n_{k}}-z\right\rangle-\frac{\beta_{n_{k}}}{\left(\alpha_{n_{k}}\right.} \\
& \sum_{n_{k}}\left(\alpha_{i-1}-\alpha_{i}\left\langle\left(I-V_{i}\right) y_{n_{k}}, x_{n_{k}}-S x_{n_{k}}\right\rangle\right. \\
& -\frac{1}{\alpha_{n_{k}}}\left\|u_{n_{k}-1}-u_{n_{k}}\right\|\left\|u_{n_{k}}-P_{C}\left[u_{n_{k}}\right]\right\| \\
& -\frac{\left(1-\alpha_{n_{k}}\right) \beta_{n_{k}}}{\alpha_{n_{k}}}\left\langle(I-S) z, x_{n_{k}}-z\right\rangle .
\end{aligned}
$$

Since $v_{n} \rightarrow 0,\left(I-V_{i}\right) y_{n} \rightarrow 0$ for all $i \geq 1$, and $\| u_{n}-$ $u_{n-1} \| / \alpha_{n} \rightarrow 0$, letting $k \rightarrow \infty$ in (44), we obtain

$$
\left\langle(I-f) x^{*}, x^{*}-z\right\rangle \leq-\tau\left\langle(I-S) z, x^{*}-z\right\rangle, \quad \forall z \in \mathcal{F} .
$$

Since (49) has the unique solution, it follows that $\omega_{w}\left(x_{n}\right)=\left\{x^{*}\right\}$. Since every weak cluster point of $\left\{x_{n}\right\}$ is also a strong cluster point, we conclude that $x_{n} \rightarrow x^{*}$ as $n \rightarrow \infty$. This completes the proof.

If we take $T_{i}=T$, for all $i \geq 1$, where $T: C \rightarrow C$ is a $k$-strict pseudo-contraction mapping in Theorem 1 , then we get the following result:

Corollary 2. Let $C$ be a nonempty closed and convex subset of a real Hilbert space $H$. Let $f: C \rightarrow H$ be a $\rho$-contraction mapping, $S: C \rightarrow H$ be a nonexpansive mapping and $T: C \rightarrow C$ be a $k$-strict pseudo-contraction mapping such that $F(T) \neq \emptyset$. Let $x_{1} \in C$ and define the sequence $\left\{x_{n}\right\}$ by

$$
\left\{\begin{array}{l}
y_{n}=P_{C}\left[\beta_{n} S x_{n}+\left(1-\beta_{n}\right) x_{n}\right], \\
x_{n+1}=P_{C}\left[\alpha_{n} f\left(x_{n}\right)+\left(1-\alpha_{n}\right) V y_{n}\right], \quad \forall n \geq 1,
\end{array}\right.
$$

where $V=k I+(1-k) T,\left\{\alpha_{n}\right\} \subset(0,1)$ and $\left\{\beta_{n}\right\} \subset(0,1)$ are sequences satisfying the conditions (H2) with $\tau=0$,
(H3), either (H4) and (H5), or (H6) and (H7). Then the sequence $\left\{x_{n}\right\}$ converges strongly to a point $z \in F(T)$, which is the unique solution of the variational inequality:

$$
\langle(I-f) z, x-z\rangle \geq 0, \quad \forall x \in F(T) .
$$

Taking $k_{i}=0$, for all $i \geq 1$ in Theorem 1 , then we get the following result:

Corollary 3. Let $C$ be a nonempty closed and convex subset of a real Hilbert space $H$. Let $f: C \rightarrow H$ be a $\rho$-contraction mapping, $S: C \rightarrow H$ be a nonexpansive mapping and $\left\{T_{i}\right\}_{i=1}^{\infty}: C \rightarrow C$ be a countable family of nonexpansive mappings and $\mathcal{F}=\cap_{i=1}^{\infty} F\left(T_{i}\right) \neq \emptyset$. Let $\alpha_{0}=1, x_{1} \in C$ and define the sequence $\left\{x_{n}\right\}$ by

$$
\left\{\begin{array}{l}
y_{n}=P_{C}\left[\beta_{n} S x_{n}+\left(1-\beta_{n}\right) x_{n}\right], \\
x_{n+1}=P_{C}\left[\alpha_{n} f\left(x_{n}\right)+\sum_{i=1}^{n}\left(\alpha_{i-1}-\alpha_{i}\right) T_{i} y_{n}\right], \quad \forall n \geq 1,
\end{array}\right.
$$

where $\left\{\alpha_{n}\right\} \subset(0,1)$ and $\left\{\alpha_{n}\right\}$ is a strictly decreasing sequence, $\left\{\beta_{n}\right\} \subset(0,1)$ and $\left\{\alpha_{n}\right\}$ and $\left\{\beta_{n}\right\}$ are sequences satisfying the conditions (H2) with $\tau=0$, (H3), either (H4) and (H5), or (H6) and (H7). Then the sequence $\left\{x_{n}\right\}$ converges strongly to a point $z \in \mathcal{F}$, which is the unique solution of the variational inequality:

$$
\langle(I-f) z, x-z\rangle \geq 0, \quad \forall x \in \mathcal{F} .
$$

If we take $k=0$ in Corollary 2, then we get the following result:

Corollary 4. Let $C$ be a nonempty closed and convex subset of a real Hilbert space H. Let $f: C \rightarrow H$ be a $\rho$-contraction mapping, $S: C \rightarrow H$ be a nonexpansive mapping and $T: C \rightarrow C$ be a nonexpansive mapping such that $F(T) \neq \emptyset$. Let $x_{1} \in C$ and define the sequence $\left\{x_{n}\right\}$ by

$$
\left\{\begin{array}{l}
y_{n}=P_{C}\left[\beta_{n} S x_{n}+\left(1-\beta_{n}\right) x_{n}\right], \\
x_{n+1}=P_{C}\left[\alpha_{n} f\left(x_{n}\right)+\left(1-\alpha_{n}\right) T y_{n}\right], \quad \forall n \geq 1,
\end{array}\right.
$$

where $\left\{\alpha_{n}\right\} \subset(0,1),\left\{\beta_{n}\right\} \subset(0,1)$ and $\left\{\alpha_{n}\right\}$ and $\left\{\beta_{n}\right\}$ are sequences satisfying the conditions (H2) with $\tau=0$, (H3), either (H4) and (H5), or (H6) and (H7). Then the sequence $\left\{x_{n}\right\}$ converges strongly to a point $z \in F(T)$, which is the unique solution of the variational inequality:

$$
\langle(I-f) z, x-z\rangle \geq 0, \quad \forall x \in F(T) .
$$

If we take $T_{i}=T$, for all $i \geq 1$, where $T: C \rightarrow C$ is a $k$-strict pseudo-contraction mapping in Theorem 2, then we obtain the following result: 
Corollary 5. Let $C$ be a nonempty closed and convex subset of a real Hilbert space H. Let $f: C \rightarrow H$ be a $\rho$-contraction mapping, $S: C \rightarrow C$ be a nonexpansive mapping and $T: C \rightarrow C$ be a k-strict pseudo-contraction mapping and $\mathcal{F}=F(T) \neq \emptyset$. Let $x_{1} \in C$ and define the sequence $\left\{x_{n}\right\}$ by

$$
\left\{\begin{array}{l}
y_{n}=\beta_{n} S x_{n}+\left(1-\beta_{n}\right) x_{n}, \\
x_{n+1}=P_{C}\left[\alpha_{n} f\left(x_{n}\right)+\left(1-\alpha_{n}\right) V y_{n}\right], \quad \forall n \geq 1,
\end{array}\right.
$$

where $V=k I+(1-k) T,\left\{\alpha_{n}\right\} \subset(0,1),\left\{\beta_{n}\right\} \subset(0,1)$ and $\left\{\alpha_{n}\right\}$ and $\left\{\beta_{n}\right\}$ are sequences satisfying the conditions (H2) with $\tau \in(0, \infty)$, (H3), (H8) and (H9). Then the sequence $\left\{x_{n}\right\}$ converges strongly to a point $x^{*} \in \mathcal{F}$, which is the unique solution of the variational inequality:

$$
\left\langle\frac{1}{\tau}(I-f) x^{*}+(I-S) x^{*}, x-x^{*}\right\rangle \geq 0, \quad \forall x \in \mathcal{F} .
$$

If we take $k_{i}=0$, for all $i \geq 1$ in Theorem 2 , then we get the following result:

Corollary 6. Let $C$ be a nonempty closed and convex subset of a real Hilbert space H. Let $f: C \rightarrow H$ be a $\rho$-contraction mapping, $S: C \rightarrow C$ be a nonexpansive mapping and $\left\{T_{i}\right\}_{i=1}^{\infty}: C \rightarrow C$ be a countable family of nonexpansive mappings and $\mathcal{F}=\cap_{i=1}^{\infty} F\left(T_{i}\right) \neq \emptyset$. Let $\alpha_{0}=1, x_{1} \in C$ and define the sequence $\left\{x_{n}\right\}$ by

$$
\left\{\begin{array}{l}
y_{n}=\beta_{n} S x_{n}+\left(1-\beta_{n}\right) x_{n}, \\
x_{n+1}=P_{C}\left[\alpha_{n} f\left(x_{n}\right)+\sum_{i=1}^{n}\left(\alpha_{i-1}-\alpha_{i}\right) T_{i} y_{n}\right], \quad \forall n \geq 1,
\end{array}\right.
$$

where $\left\{\alpha_{n}\right\} \subset(0,1)$ and $\left\{\alpha_{n}\right\}$ is a strictly decreasing sequence, $\left\{\beta_{n}\right\} \subset(0,1)$ and $\left\{\alpha_{n}\right\}$ and $\left\{\beta_{n}\right\}$ are sequences satisfying the conditions (H2) with $\tau \in(0, \infty)$, (H3), (H8) and (H9). Then the sequence $\left\{x_{n}\right\}$ converges strongly to a point $x^{*} \in \mathcal{F}$, which is the unique solution of the variational inequality:

$$
\left\langle\frac{1}{\tau}(I-f) x^{*}+(I-S) x^{*}, x-x^{*}\right\rangle \geq 0, \quad \forall x \in \mathcal{F} .
$$

If $k=0$ in Corollary 5, then we get the following Corollary:

Corollary 7. Let $C$ be a nonempty closed and convex subset of a real Hilbert space $H$. Let $f: C \rightarrow H$ be a $\rho$-contraction mapping, $S, T: C \rightarrow C$ be nonexpansive mappings and $\mathcal{F}=F(T) \neq \emptyset$. Let $x_{1} \in C$ and define the sequence $\left\{x_{n}\right\}$ by

$$
\left\{\begin{array}{l}
y_{n}=\beta_{n} S x_{n}+\left(1-\beta_{n}\right) x_{n}, \\
x_{n+1}=P_{C}\left[\alpha_{n} f\left(x_{n}\right)+\left(1-\alpha_{n}\right) T y_{n}\right], \quad \forall n \geq 1,
\end{array}\right.
$$

where $\left\{\alpha_{n}\right\} \subset(0,1),\left\{\beta_{n}\right\} \subset(0,1)$ and $\left\{\alpha_{n}\right\}$ and $\left\{\beta_{n}\right\}$ are sequences satisfying the conditions (H2) with $\tau \in(0, \infty)$, (H3), (H8) and (H9). Then the sequence $\left\{x_{n}\right\}$ converges strongly to a point $x^{*} \in \mathcal{F}$, which is the unique solution of the variational inequality:

$$
\left\langle\frac{1}{\tau}(I-f) x^{*}+(I-S) x^{*}, x-x^{*}\right\rangle \geq 0, \quad \forall x \in \mathcal{F} .
$$

Remark 2. Prototypes for the iterative parameters are, for example, $\alpha_{n}=n^{-\theta}$ and $\beta_{n}=n^{-\omega}$ (with $\theta, \omega>0$ ). Since $\left|\alpha_{n}-\alpha_{n-1}\right| \approx n^{-\theta}$ and $\left|\beta_{n}-\beta_{n-1}\right| \approx n^{-\omega}$, it is not difficult to prove that $(H 8)$ is satisfied for $0<\theta, \omega<1$ and (H9) is satisfied if $\theta+\omega \leq 1$.

Remark 3. Theorem 1 and Theorem 2 extend and improve the result of $\mathrm{Gu}$ et al. (2011) from the countable family of nonexpansive mappings to more general the countable family of strictly pseudo contraction mappings.

\section{Competing interests}

The authors declare that they have no competing interests.

\section{Authors' contributions}

All authors contributed equally and significantly in writing this paper. All authors read and approved the final manuscript.

\section{Acknowledgements}

The authors would like to thank the Higher Education Research Promotion and National Research University Project of Thailand's Office of the Higher Education Commission for financial support (under NRU-CSEC Project no. 56000508)

\section{Author details}

${ }^{1}$ Department of Mathematics, Faculty of Science, King Mongkut's University of Technology Thonburi (KMUTT), 126 Pracha Uthit Road, Bang Mod, Thrung Khru, Bangkok 10140, Thailand. ${ }^{2}$ Department of Mathematics and Statistics, Faculty of Science, Valaya Alongkorn Rajabhat University under the Royal Patronage, Pahonyotin Road, Pathumtani 13180, Thailand

Received: 29 July 2013 Accepted: 19 September 2013 Published: 17 October 2013

\section{References}

Acedo GL, Xu HK (2007) Iterative methods for strictly pseudo-contractions in hilbert space. Nonlinear Anal 67:2258-2271

Baiocchi C, Capelo A (1984) Variational and quasivariational inequalities: applications to free boundary problems. A Wiley-Interscience Publication, New York

Browder FE (1976) Nonlinear operators and nonlinear equations of evolution in banach spaces. Proc Symposia in Pure Math 18:78-81

Cho YJ, Kang SM, Qin X (2009) Some results on k-strictly pseudo-contractive mappings in hilbert spaces. Nonlinear Anal 70:1956-1964

Cianciaruso F, Marino G, muglia L, Yao Y (2009) On a two-steps algorithm for hierarchical fixed point problems and variational inequalities. J Inequalities and Applications 2009:13

Giannessi F, Maugeri A (1995) Variational inequalities and network equilibrium problems. Plenum Press, New York 
Gu G, Wang S, Cho YJ (2011) Strong convergence algorithms for hierarchical fixed points problems and variational inequalities. J Appl Math 2011:17

Jung JS (2010) Strong convergence of iterative methods for k-strictly pseudo-contractive mappings in hilbert spaces. Appl Math Comput 215: 3746-3753

Jung JS (2011) Some results on a general iterative method for k-strictly pseudo-contractive mappings. Fixed Point Theo and Appl 24:11

Kinderlehrer D, Stampacchia G (1980) An Introduction to Variational Inequalities and Their Applications. Academic Press, New York

Mainge PE, Moudafi A (2007) Strong convergence of an iterative method for hierarchical fixed-point problems. Pacific J Optimization 3(3):529-538

Marino G, Xu HK (2006) A general iterative method for nonexpansive mappings in hilbert spaces. J Math Anal Appl 318:43-52

Marino G, Xu HK (2011) Explicit hierarchical fixed point approach to variational inequalities. J Optimization Theory and Applications 149:61-78

Moudafi A (2000) Viscosity approximation methods for fixed-points problems. J Math Anal Appl 241:46-55

Moudafi A (2007) Krasnoselski-mann iteration for hierarchical fixed-point problems. Inverse Problems 23(4):1635-1640

Noor MA (2004) Some developments in general variational inequalities. Appl Math and Comput 152:199-277

Noor MA (2009) Extended general variational inequalities. Nonlinear Anal 22: 182-186

Noor MA, Noor KI, Al-Said E (2012a) Iterative methods for solving nonconvex equilibrium variational inequalities. Appl Math \& Info Sci 6:65-69

Noor MA, Noor Kl, Huang ZY, Al-Said E (2012b) Implicit schemes for solving extended general nonconvex vatiational inequalities. J Appl Math 2012:10

Xu HK (2002) Iterative algorithms for nonlinear operator. J London Math Soc $66: 240-256$

Xu HK (2004) Viscosity approximation methods for nonexpansive mappings. $J$ Math Anal Appl 298:279-291

Yao Y, Cho YJ, Liou YC (2010) Iterative algorithms for hierarchical fixed points problems and variational inequalities. Math and Comput Modell 52(9-10): 1697-1705

Zhou H (2008) Convergence theorems of fixed points for k-strict pseudo-contractions in hilbert spaces. Nonlinear Anal 69:456-462

doi:10.1186/2193-1801-2-540

Cite this article as: Chamnarnpan et al: Hierarchical fixed points of strictly pseudo contractive mappings for variational inequality problems. SpringerPlus 2013 2:540.

\section{Submit your manuscript to a SpringerOpen ${ }^{\circ}$ journal and benefit from:}

- Convenient online submission

- Rigorous peer review

- Immediate publication on acceptance

- Open access: articles freely available online

- High visibility within the field

- Retaining the copyright to your article

Submit your next manuscript at $\boldsymbol{\wedge}$ springeropen.com 